\title{
Finite element modeling of a generic stemless hip implant design in comparison with conventional hip implants
}

\begin{abstract}
In this study, the finite element modeling and comparison of the stress and strain analyses were carried out for three different structures that are intact bone, stemless implant and stemmed one. Currently proposed stemless design studied here is the generic concept of stemless implant. This generic stemless implant reconstruction was numerically compared to the conventional stemmed implant and also to the intact bone as control solution. Two loading conditions were applied to the most proximal part of the models, while the most distal part was fixed for all degrees of freedom. The models were divided into two regions and studied along two paths of medial and lateral aspect. The results of this study showed that the stemless implant had less deviation from the control solution of the bone in all regions and in both loading conditions, comparing to the large deviation of the stemmed implant from the intact bone. However, it was shown that the fixation of this type of implant and its effect on sub-trochanter region must be carefully considered for designing the final product of any specific design of stemless implant.
\end{abstract}

Keyword: Hip implant, Conventional implant, Stemless and finite element analysis 